\title{
Visualizing Communities and Centralities from Encounter Traces
}

\author{
Eiko Yoneki \\ University of Cambridge, Computer Laboratory \\ Cambridge CB3 0FD, United Kingdom \\ eiko.yoneki@cl.cam.ac.uk
}

\begin{abstract}
We have previously demonstrated that information about social relationships can yield improved performance when it is used to control epidemic forwarding [7]. We believe that extensive work to model human connectivity - incorporating notions of community and interaction 'weight' - is required if we are to understand this phenomenon and build networks that capitalize on it. This paper describes a visualization of detected community structures uncovered by different methods from human encounter traces. We focus on extracting information related to levels of clustering, network transitivity, and strong community structure. The position change of hub nodes within the network is also visualized.
\end{abstract}

\section{Categories and Subject Descriptors}

C.2.4 [Computer Systems Organization]: Computer Communication Networks-Distributed Systems; I.6 [Computing Methodologies]: Simulation and Modeling

\section{General Terms}

Measurement, Experimentation, Algorithms

\section{Keywords}

Delay Tolerant Networks, Network Measurement, Social Networks, Centrality, Clustering

\section{INTRODUCTION}

As mobile, network-enabled devices become more common, we gain the opportunity to exploit the dynamic networks that form among them. However, these networks pose significant challenges: the devices are sparsely distributed, and the network often becomes partitioned due to geographical separation or node movement. The emergence of Delay Tolerant Networks (DTNs) has culminated in a new generation of wireless networking addressing such network environments. New communication paradigms, which use dynamic interconnectedness as people encounter each other leading towards a world where digital traffic flows in opportunistic manner. We focus on human-to-human (H2H) communica-

Permission to make digital or hard copies of all or part of this work for personal or classroom use is granted without fee provided that copies are not made or distributed for profit or commercial advantage and that copies bear this notice and the full citation on the first page. To copy otherwise, to republish, to post on servers or to redistribute to lists, requires prior specific permission and/or a fee.

CHANTS'08, September 15, 2008, San Francisco, California, USA.

Copyright 2008 ACM 978-1-60558-186-6/08/09 ...\$5.00. tion among such networks that exhibit the characteristics of social networks.

We have previously demonstrated that information about social relationships can yield improved performance when it is used to control epidemic forwarding [7]. This is particularly useful when applied to Pocket Switched Networks (PSNs): a subclass of DTNs, in which each node is a mobile device carried by a human being. Furthermore, we have shown that it is possible to infer social networks from human connectivity traces. During this work, we realized that further understanding of network models is essential, because the properties of human contact networks - such as community and weight of interactions - are important aspects.

In this paper, we show a visualization of detected community structures using different community detection algorithms from human encounter traces especially a snapshot of hierarchical community detection results. We focus on extracting information related to levels of clustering, network transitivity, and strong community structure. The position change hub nodes along changing community formation is also visualized.

\section{INFERRING HUMAN COMMUNITIES}

People inherently form groups, yielding social structures in which prominent patterns or information flow can be observed. Community detection in complex networks has attracted a lot of attention in recent years. In the Internet, community structures correspond to autonomous systems. It is crucial to construct efficient algorithms for identifying the community structure in a generic network. Many community detection methods have been proposed and examined in the literature (see the recent review papers by Newman [9] and Danon et al. [2]). We have shown various community detection mechanisms [7] which can be applied to human connectivity traces in both a centralized and a decentralized way. The following summarizes the outline of algorithms (see [7] for further details).

- K-Clique: Palla et al. define a community as a union of all $k$-cliques (complete sub-graphs of size $k$ ) that can be reached from each other through a series of adjacent $k$-cliques, where two $k$-cliques are said to be adjacent if they share $k-1$ nodes. An advantage of this approach is that it allows overlapping communities, which is useful as, in human society, one person may belong to multiple communities.

- Weighted Networks Analysis by Newman [8] can work on weighted graphs built from encounter trace. A weighted graph can be converted into a multi-graph 

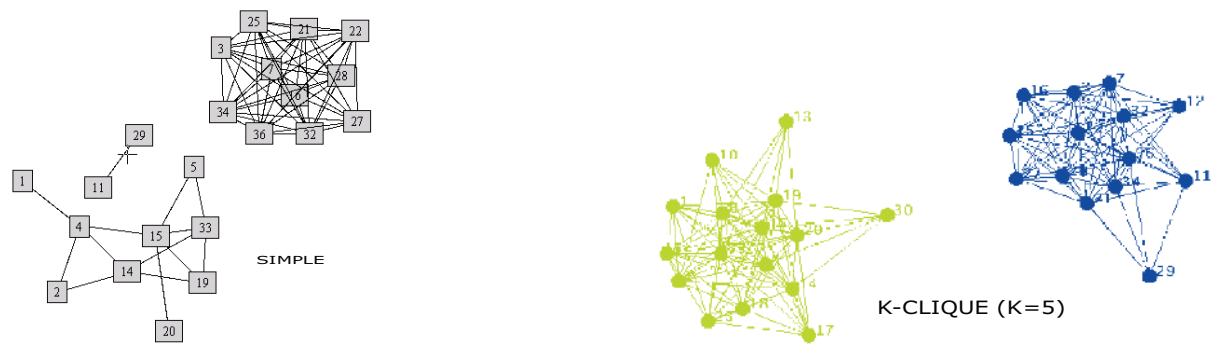

Figure 1: Communities - SIMPLE and K-Clique (CAM trace)

with many unit edges.

- Fiedler Clustering: The eigenvector for the nonzero smallest eigenvalue of a Laplacian matrix is called the Fiedler vector [5]. This vector can be used for decomposing graphs into structural components.

As self-organizing networks, we would also ask whether the mobile devices can sense and detect their own local communities instead of relying on a centralized server, which leads to the area of distributed community detection. We have shown three different algorithms named SIMPLE, $K$ CLIQUE and MODULARITY, for distributed community detection, and they proved that the detecting accuracy can be up to $85 \%$ of the centralized $K$-CLIQUE algorithm. See [7] for the details.

\section{VISUALIZING COMMUNITIES}

The quantitative understanding of human dynamics is difficult and has not yet been explored in depth. The emergence of human interaction traces from online and pervasive environments allows us to understand details of human activities. For example, the Reality Mining project [4] collected proximity, location and activity information, with nearby nodes being discovered through periodic Bluetooth scans and location information from cell tower IDs. Several other groups have performed similar studies. Most of these [4] [3] use Bluetooth to measure device connectivity, while others [6] rely on WiFi. The duration of experiments varies from 2 days to over one year, and the numbers of participants vary. We have analyzed various traces from Crawdad database [1] listed below and Table 1 summaries the configuration.

We show a snapshot of visualization in Fig. 1 and Fig. 2, where detected communities with different algorithms are depicted. Fig. 1 shows the community structures of the CAM trace, where distinct two communities are observed. The CAM trace contains the encounter log of the first and second year undergraduate students. Both SIMPLE from the distributed detection and $K$-Clique depict similar results. Fig. 2 demonstrates hierarchical structures of communities by Fiedler Clustering. The Cambridge result shows two clusters clearly.

\section{INFLUENCE OF HUB NODES}

We have defined hubs based on the following centralities (see [11] for further detail). Hub nodes are influential nodes within the network.

\begin{tabular}{|c|r|r|r|r|r|}
\hline Experimental data set & MIT & UCSD & CAM & INFC06 & BATH \\
\hline Device & Phone & PDA & iMote & iMote & PC \\
Network type & Bluetooth & WiFi & Bluetooth & Bluetooth & Bluetooth \\
Duration (days) & 246 & 77 & 11 & 3 & 5.5 \\
Granularity (seconds) & 300 & 600 & 120 & 120 & Continuous \\
Number of Experimental Devices & 97 & 274 & 36 & 78 & 7431 \\
\hline
\end{tabular}

Table 1: Characteristics of the experiments
- DEGREE Hub: The total degree of each node over the entire duration of the trace indicates the popularity of the node (Degree Centrality).

- RANK Hub: The frequency that a node is used to relay data to other nodes indicates the Betweenness Centrality. We simulated flooding over the temporal graph extracted from the trace and counted the number of times each node is used for relaying the data.

- CROSS Hub: The appearance of a node at different locations indicates that it has Mobility Centrality.

We visualize the position of hub nodes within the network leading to discover the characteristics of hub nodes. Fig. 3 depicts network evolution over a period 15 minutes in the UCSD trace (taken from our visualization work [10]). The network exhibits a small-world-like formation at first, which breaks down into two groups, each forming a star topology. The node 35 with dotted circle positions always center of a clique, while the node 19 with double circles takes place at the edge of a cluster.

\section{CONCLUSIONS}

In this paper, we show visualization of detected community structures uncovered by different community detection algorithms from human encounter traces. The movement of hub nodes within the network is also visualized. Visualizing dynamic node behavior gives an aid to understand complex network structure and network transitivity. We are currently working on integrating location information in visualization.

\section{REFERENCES}

[1] D. College. http://crawdad.cs.dartmouth.edu/index.php, 2007.

[2] L. Danon, J. Duch, A. Diaz-Guilera, and A. Arenas. Comparing community structure identification, 2005.

[3] Haggle Project, http://www.haggleproject.org, 2008.

[4] N. Eagle et al. Reality mining: sensing complex social systems. Personal and Ubiquitous Computing, 10(4):255-268, 2006.

[5] M. Fiedler. A property of eigenvectors of nonnegative symmetric matrices and its application to graph theory. Czech Math J., 25, 1975.

[6] T. Henderson et al. The changing usage of a mature campus-wide wireless network. In Proc. Mobicom, 2004.

[7] P. Hui et al. BUBBLE Rap: Social Based Forwarding in Delay Tolerant Networks. In proc. MobiHoc, 2008.

[8] M. Newman. Analysis of weighted networks. Physical Review E, 70:056131, 2004.

[9] M. Newman. Detecting community structure in networks. Eur. Phys. J. B, 38:321-330, 2004.

[10] E. Yoneki et al. Visualizing Community Detection in Opportunistic Networks. In ACM CHANTS, 2007.

[11] E. Yoneki et al. Distinct Types of Hubs in Human Dynamic Network. In EuroSys Socnet, 2008. 

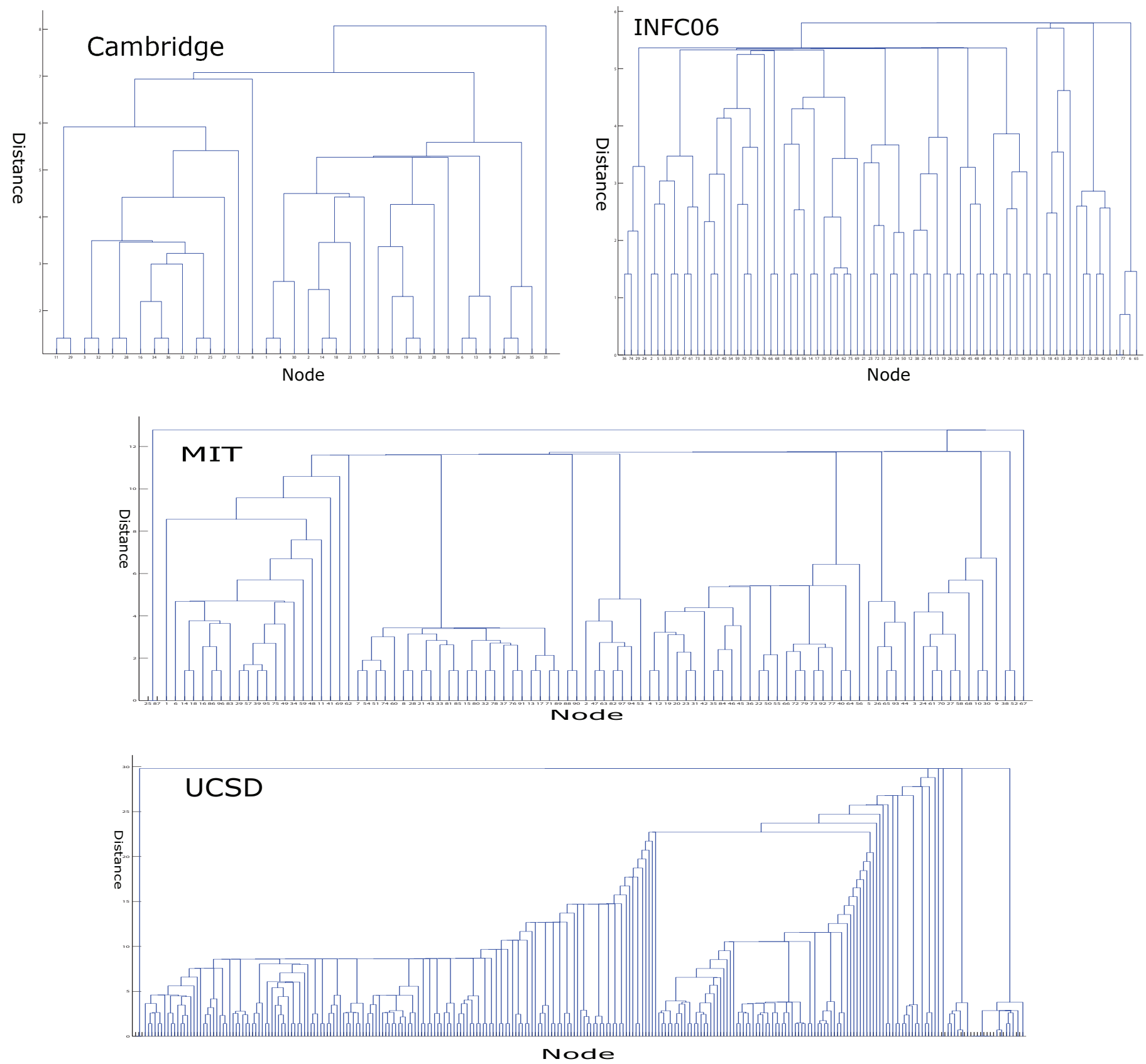

Figure 2: Hierarchical Community Structure - Fiedler Clustering
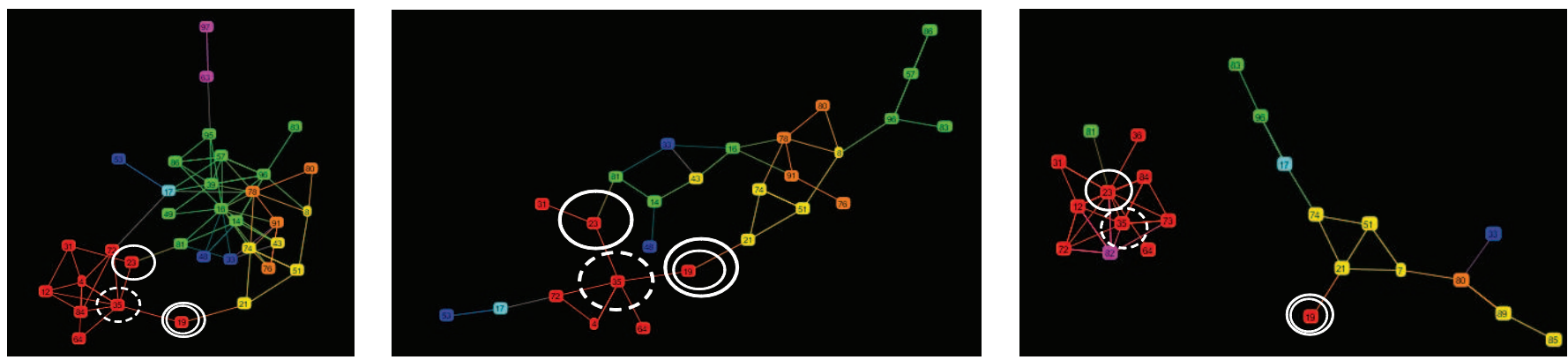

Figure 3: Position Change of Hub Nodes (UCSD Trace) 